\title{
Population-scale Longitudinal Mapping of COVID-19 Symptoms, Behavior, and Testing Identifies Contributors to Continued Disease Spread in the United States
}

William E. Allen ${ }^{1,2,3 * \dagger}$, Han Altae-Tran ${ }^{1,3,4 *}$, James Briggs ${ }^{1,3,5 *}$, Xin Jin ${ }^{1,2,3 *}$, Glen McGee ${ }^{1,6 *}$, Andy Shi ${ }^{1,6 *}$, Rumya Raghavan ${ }^{1,3,7}$, Mireille Kamariza ${ }^{1,2,3}$, Nicole Nova ${ }^{1,8}$, Albert Pereta ${ }^{1}$, Chris

Danford $^{1}$, Amine Kamel ${ }^{1}$, Patrik Gothe ${ }^{1}$, Evrhet Milam ${ }^{1}$, Jean Aurambault ${ }^{1}$, Thorben Primke ${ }^{1}$, Weijie Li ${ }^{1}$, Josh Inkenbrandt ${ }^{1}$, Tuan Huynh ${ }^{1}$, Evan Chen ${ }^{1}$, Christina Lee ${ }^{1}$, Michael Croatto ${ }^{1}$, Helen Bentley ${ }^{1}$, Wendy Lu ${ }^{1}$, Robert Murray ${ }^{1}$, Mark Travassos ${ }^{1,9}$, Brent A. Coull ${ }^{6}$, John Openshaw ${ }^{1,10}$, Casey S. Greene ${ }^{1,11}$, Ophir Shalem ${ }^{1,12}$, Gary King ${ }^{1,13}$, Ryan Probasco ${ }^{1}$, David R. Cheng $^{1}$, Ben Silbermann ${ }^{1}$, Feng Zhang ${ }^{1,3,4,14,15,16} \uparrow$, Xihong Lin ${ }^{1,3,6,17} \uparrow$

* These authors contributed equally

† Correspondence to: weallen@fas.harvard.edu, zhang_f@mit.edu, xlin@hsph.harvard.edu

${ }^{1}$ The How We Feel Project

${ }^{2}$ Society of Fellows, Harvard University, Cambridge, MA, USA

${ }^{3}$ Broad Institute of MIT and Harvard, Cambridge, MA, USA

${ }^{4}$ Department of Biological Engineering, Massachusetts Institute of Technology, Cambridge, MA, USA

${ }^{5}$ Schmidt Science Fellow

${ }^{6}$ Department of Biostatistics, Harvard T.H. Chan School of Public Health, Boston, MA

${ }^{7}$ Health Sciences and Technology Program, Massachusetts Institute of Technology and Harvard Medical School, Cambridge, MA

${ }^{8}$ Department of Biology, Stanford University, Stanford, CA

${ }^{9}$ Center for Vaccine Development and Global Health, University of Maryland School of Medicine, Baltimore, MD

${ }^{10}$ Division of Infectious Diseases and Geographic Medicine, Department of Medicine, Stanford University School of Medicine, Stanford, CA

${ }^{11}$ Department of Systems Pharmacology and Translational Therapeutics, University of Pennsylvania Perelman School of Medicine, Philadelphia, PA

${ }^{12}$ Department of Genetics, University of Pennsylvania Perelman School of Medicine, Philadelphia, PA

${ }^{13}$ Albert J. Weatherhead III University Professor, Institute for Quantitative Social Sciences, Harvard University, Cambridge, MA

${ }^{14} \mathrm{McGovern}$ Institute for Brain Research, Massachusetts Institute of Technology, Cambridge, MA, USA

${ }^{15}$ Department of Brain and Cognitive Sciences, Massachusetts Institute of Technology, Cambridge, MA, USA

${ }^{16}$ Howard Hughes Medical Institute, Chevy Chase, MD, USA

${ }^{17}$ Department of Statistics, Harvard University, Cambridge, MA, USA 
medRxiv preprint doi: https://doi.org/10.1101/2020.06.09.20126813; this version posted June 11, 2020. The copyright holder for this preprint (which was not certified by peer review) is the author/funder, who has granted medRxiv a license to display the preprint in perpetuity.

\section{Summary Paragraph}

Despite social distancing and shelter-in-place policies, COVID-19 continues to spread in the United States. A lack of timely information about factors influencing COVID-19 spread and testing has hampered agile responses to the pandemic. We developed How We Feel, an extensible web and mobile application that aggregates self-reported survey responses, to fill gaps in the collection of COVID-19-related data. How We Feel collects longitudinal and geographically localized information on users' health, behavior, and demographics. Here we report results from over 500,000 users in the United States from April 2, 2020 to May 12, 2020. We show that selfreported surveys can be used to build predictive models of COVID-19 test results, which may aid in identification of likely COVID-19 positive individuals. We find evidence among our users for asymptomatic or presymptomatic presentation, as well as for household and community exposure, occupation, and demographics being strong risk factors for COVID-19. We further reveal factors for which users have been SARS-CoV-2 PCR tested, as well as the temporal dynamics of selfreported symptoms and self-isolation behavior in positive and negative users. These results highlight the utility of collecting a diverse set of symptomatic, demographic, and behavioral selfreported data to fight the COVID-19 pandemic.

\section{Main Text}

The rapid global spread of severe acute respiratory syndrome coronavirus 2 (SARS-CoV-2), the novel virus causing coronavirus disease 2019 (COVID-19) ${ }^{1-3}$, has created an unprecedented public health emergency. In the United States, efforts to slow the spread of disease have included, to varying extents, social distancing, home-quarantine and treating infected patients, mandatory facial covering, closure of schools and non-essential businesses, and testing-trace-isolate measures ${ }^{4,5}$. The COVID-19 pandemic and ensuing response has produced a concurrent economic crisis of a scale not seen for nearly a century ${ }^{6}$, exacerbating the effect of the pandemic on different socioeconomic groups and producing adverse health outcomes beyond COVID-19. As a result, there is currently intense pressure to safely wind down these measures. Yet, in spite of widespread lockdowns and social distancing throughout the US, many states continue to exhibit steady increases in the number of cases ${ }^{7}$. In order to understand where and why the disease continues to spread, there is a pressing need for real-time individual-level data on COVID-19 infections and tests, as well as on the behavior, exposure, and demographics of individuals at the population scale with granular location information. These data will allow medical professionals, public health officials, and policy makers to understand the effects of the pandemic on society, tailor intervention measures, efficiently allocate testing resources, and address disparities.

One approach to collecting this type of data on a population scale is to use web- and mobile-phone based surveys that enable large-scale collection of self-reported data. Previous studies, such as FluNearYou, have demonstrated the potential for using online surveys for disease surveillance ${ }^{8}$. 
medRxiv preprint doi: https://doi.org/10.1101/2020.06.09.20126813; this version posted June 11, 2020. The copyright holder for this preprint (which was not certified by peer review) is the author/funder, who has granted medRxiv a license to display the preprint in perpetuity.

All rights reserved. No reuse allowed without permission.

Since the start of the COVID-19 pandemic, several different applications have been launched throughout the world to collect COVID-19 symptoms, testing, and contact-tracing information ${ }^{9}$. Studies in the UK and Israel have reported large cohorts of users and demonstrated some ability to detect and predict the spread of disease ${ }^{10-12}$. Existing tools, however, focus primarily on COVID-19 symptoms. There is a strong need to investigate exposure, demographic and behavioral factors that affect the chain of transmission, understand the factors for who have been tested, and study the degree of presence of asymptomatic, presymptomatic, mildly symptomatic cases ${ }^{13}$.

To overcome these limitations, we developed How We Feel (HWF, http://www.howwefeel.org) (Fig. 1a-d), a web and mobile-phone application for collecting de-identified self-reported COVID19-related data. Rather than targeting suspected COVID-19 patients or existing study cohorts, HWF aims to collect data from users representing the population at large. Users are asked to share information on demographics (gender, age, race/ethnicity, household structure, ZIP code), COVID-19 exposure, and pre-existing medical conditions. They then self-report daily how they feel (well or not well), any symptoms they may be experiencing, test results, behavior (e.g., use of face coverings), and sentiment (e.g., feeling safe to go to work) (Fig. 1c, Extended Data Fig. 1). To protect privacy, users are not identifiable beyond a randomly-generated number that links repeated logins on the same device. A key feature of the app is the ability to rapidly release revised versions of the survey as the pandemic evolves. In the first month of operation, we released three iterations of the survey with increasingly expanded sets of questions (Fig. 1b).

The app was launched on April 2, 2020 in the United States. As of May 12, 2020, the app had 502,731 users in the United States, with 3,661,716 total responses (Fig. 1b) (Extended Data Table 1). $74 \%$ of users responded on multiple days, with an average of 7 responses per user (Extended Data Fig. 2). Each day, 5\% of users who accessed the app reported feeling unwell (Fig. 1b). The user base was distributed across all 50 states and several US territories, with the largest numbers of users in more populous states such as California, Texas, Florida, and New York (Fig. 1d). Connecticut had the largest number of users per state, as the result of a partnership with the Connecticut state government (Fig. 1d). Users were required to be 18 years of age or older and were 42 years old on average (mean: 42.0; SD: 16.3), including 18.4\% in the bracket of $60+$, which has experienced the highest mortality rate from COVID-19 (Fig. 1e) ${ }^{14,15}$. Users were primarily female (82.7\%) (Fig. 1f) and white (75.5\%, excluding 20.3\% with missing data) (Fig. 1g).

A major ongoing problem in the US is the overall lack of testing across the country ${ }^{16}$ and disparities in test accessibility, infection rates, and mortality rates in different regions and communities ${ }^{17,18}$. In the absence of population-scale testing, it will be critical during a reopening to allocate limited testing resources to the groups or individuals most likely to be infected in order to track the spread of disease and break the chain of infection. We therefore first examined who in our userbase is currently receiving testing. We analyzed 4,759 users who took the Version 3 (V3) survey and who were PCR tested for SARS-CoV-2 (out of 272,392 total users) (Fig. 2a, Extended Data Fig. 3a). 
Of these, $8.8 \%$ were PCR positive. The number of tests reported by test date displays a similar trend to the estimated number of tests across the US, suggesting that our sampling captures the increase in test availability (Fig. 2a). The number of PCR tests per HWF user is highly correlated with external estimates of per-capita tests by state (Fig. 2b, Extended Data Fig. 3b, Pearson correlation 0.77$)^{19}$.

We first examined via logistic regression which factors either collected in the survey or inferred from US Census data by user ZIP code were associated with receiving a SARS-CoV-2 PCR test, regardless of test result. As expected, we observed that a higher fraction of tested users from states with higher per-capita test numbers, according to the COVID Tracking Project ${ }^{19}$ (Extended Data Fig. 3b). Healthcare workers (OR: 2.94; 95\% CI: [2.75, 3.15]) and other essential workers (OR: $1.39 ; 95 \% \mathrm{CI}:[1.28,1.52])$ were more likely to have received a PCR test compared to users who did not report those professions (Fig. 2c). Users who reported experiencing fever, cough, or loss of taste/smell (among other symptoms) had higher odds of being tested compared to users who never reported these symptoms (Fig. 2c). The majority of these symptoms are listed as common for COVID-19 cases by the Centers for Disease Control and Prevention (CDC) (Fig. 2c, starred) ${ }^{20}$. A less common symptom, reporting a tight feeling in one's chest, was also associated with receiving a PCR-based test (OR: $2.27,95 \%$ CI: $[1.93,2.66])$. These results suggest that the most commonly reported symptoms are being used as screening criteria for determining who receives a test, potentially missing individuals with less common symptom presentations. This group could include those who are at high risk for infection but do not meet the testing eligibility criteria.

To obtain a global view of self-reported symptom patterns, we applied an unsupervised manifold learning algorithm to visualize how symptoms were correlated across users (Methods). As expected, we found that symptom presentation separated broadly by feeling well versus feeling unwell (Fig. 2d, Extended Data Fig. 4). Users who felt unwell were concentrated in a single cluster indicating similar overall symptom profiles, which was characterized by high proportions of common COVID-19 symptoms as defined by the $\mathrm{CDC}^{20}$ (Fig. 2e), and contained the vast majority of responses from users with both positive $(+)$ and negative (-) SARS-CoV-2 PCR tests (Fig. 2f). Thus COVID-19 symptoms tend to overlap with symptoms for other diseases and do not necessarily predict positive test results.

This overlap suggests that commonly used symptoms may not be sufficient criteria for evaluating COVID-19 infection. It has previously been reported that many people infected with SARS-CoV2 are asymptomatic, mildly symptomatic, or in the presymptomatic phase of their presentation ${ }^{21-}$ 23 and therefore unaware that they are infected. In our dataset, on the day of their test, most users (73\%) that tested PCR positive for SARS-CoV-2 reported feeling unwell with the common symptoms listed by the CDC (dry cough, shortness of breath, chills/shaking, fever, muscle/joint pain, sore throat, loss of taste/smell). However, $11.5 \%$ of positive users reported feeling unwell and exclusively reported symptoms not listed as common for COVID-19 by the CDC on the day 
of their test and, and 15.4\% reported feeling no symptoms at all (Fig. $2 \mathrm{~g}$ ). Because of the commonly used symptom and occupation based screening criteria for receiving a PCR test and under-testing, this total of $36.9 \%$ likely underestimates the true fraction of asymptomatic, presymptomatic, and mildly symptomatic cases, which in Wuhan, China was estimated to be $\sim 80 \%{ }^{13}$. A large number of asymptomatic cases were also observed in serological studies ${ }^{24,25}$. $48.9 \%$ of users testing negative for SARS-CoV-2 reported feeling unwell with most common COVID-19 symptoms, compared to an expected false negative rate of 20-30\% for PCR-based tests of symptomatic patients ${ }^{26}$, again suggesting symptom presentation overlap with other diseases (Fig. 2g).

We investigated the symptoms that were most predictive of COVID-19 by exploring the distribution and dynamics of symptoms in PCR test $(+)$ and $(-)$ users around the test date. PCR test $(+)$ users reported higher rate of common COVID-19 symptoms, including dry cough, fever, loss of appetite, and loss of taste and/or smell, than PCR test (-) users (Fig. 2h). Many PCR-tested users longitudinally reported symptoms in the app in an interval extending \pm 2 weeks from their test date (Extended Data Fig. 5). We used these data to examine the time course of symptoms among those who tested positive. In the days preceding a test, dry cough, muscle pain, and nasal congestion were among the most commonly reported symptoms. Reported symptoms peaked in the week following a test and declined thereafter (Fig. 2i). Taking the ratio of the symptom rates at each point in time between PCR test $(+)$ and $(-)$ users showed that the most distinguishing feature in users who tested positive was loss of taste and/or smell, as has been previously reported ${ }^{10}$ (Fig. $2 \mathrm{j})$.

We next investigated medical and demographic factors associated with testing PCR positive for acute SARS-CoV2 infection, focusing on 3,829 users who took the V3 survey within \pm 2 weeks of their reported PCR test date (315 positive, 3,514 negative) (Fig. 3a, Extended Data Tables 2-6). These users are a subset of all the users who reported taking a test in the V3 survey, as some reported test results were outside this time window. To correct for selection bias of receiving a PCR test when studying the risk factors of a positive test result, we incorporated probability of receiving PCR tests as inverse probability weights (IPW) into our logistic model of PCR test result status (+/-) (Methods $)^{27}$. As with the analysis of who received a test, the reported symptoms, loss of taste and/or smell was most strongly associated with a positive test result (OR: $33.17,95 \% \mathrm{CI}$ : [17.3, 67.94]). Other symptoms associated with testing positive included fever (OR: 6.27, 95\% CI: [2.82, 13.70]) and cough (OR: 4.45, 95\% CI: [2.83, 6.99]). Women were less likely to test positive than men (OR: 0.55, 95\% CI: [0.38, 0.80]), and both Hispanic/Latinx users (OR: 2.59, 95\% CI: $[1.67,3.97]$ ) and African-American/black users (OR: 2.35, 95\% CI: [1.25, 4.18]) were more likely to test positive than white users, highlighting potential racial disparities involved with COVID-19 infection risk. The odds of testing positive were also higher for those in high density neighborhoods (OR: 1.85, 95\% CI: [1.15, 3.07]). Healthcare workers (OR: 1.92, 95\% CI: [1.36, 2.73]) and other essential workers (OR: 1.69, 95\% CI: $[1.13,2.52])$ also had higher odds of testing positive 
medRxiv preprint doi: https://doi.org/10.1101/2020.06.09.20126813; this version posted June 11, 2020. The copyright holder for this preprint (which was not certified by peer review) is the author/funder, who has granted medRxiv a license to display the preprint in perpetuity.

All rights reserved. No reuse allowed without permission.

compared to non-essential workers. Pregnant women were substantially more likely to test positive (OR: 6.30, 95\% CI: [2.45, 14.68]). We note that this is a preliminary result based on a small sample of 48 pregnant women included in this analysis (9 test-positive, 39 test-negative) and will be reassessed as the app accumulates more users. Performing this analysis with and without correction for selection bias produced similar results (Fig. 3a).

Motivated by previous studies that reported high cluster transmissions occurred in families in China and Japan ${ }^{28,29}$, we explored household and community exposures as risk factors for users testing PCR positive. The odds of testing positive were much higher for those who reported withinhousehold exposure to someone with confirmed COVID-19 than for those who reported no exposure at all (Methods) (OR: 19.10, 95\% CI: [12.30, 30.51]) (Fig. 3a, Extended Data Table 5). This is stronger than comparing the odds of positive among those who reported exposure outside their household versus no exposure at all (OR: 3.61, 95\% CI: [2.54, 5.18]). Further, the odds of testing PCR positive are much higher for those exposed in the household versus exposed outside their household or not exposed at all, after adjusting for similar factors (OR: 10.3, 95\% CI: [6.7, 15.8]) (Extended Data Table 7). These results are consistent with previous findings that indicate a very high relative risk associated with within-household infection ${ }^{29-33}$. This is compatible with finding that other closed areas with high levels of congregation and close proximity, such as churches $^{34}$, food processing plants ${ }^{35}$, and nursing homes ${ }^{36}$, have shown similarly high risk of transmission.

Developing models to predict who is likely to be SARS-CoV-2(+) from self-reported data has been proposed as a means to help overcome testing limitations and identify disease hotspots ${ }^{10,11}$. We used data from the 3,829 users who used the app within \pm 2 weeks of their reported PCR test results to develop a set of prediction models that were able to distinguish positive and negative results with a high degree of predictive accuracy on cross-validated data (Fig. 3b). We used the machine learning method XGBoost, which outperformed other classification methods (Extended Data Fig. 6). We considered: (1) a symptoms-only model, which included only the most common COVID19 symptoms listed by the CDC; (2) an expanded model, which further incorporated other features observed in the survey; and (3) a minimal-features model, which retained only the four most predictive features (loss of taste and/or smell, exposure to someone with COVID-19, exposure in the household to someone with confirmed COVID-19, and exposure to household members with COVID-19 symptoms) (Methods, Extended Data Tables 8-11). The symptoms-only model achieved a cross validated AUC (area under the ROC curve) of 0.76 using data before and after a test, and AUC 0.69 using just the pre-test data. Expanding the set of features to include other survey questions substantially improved performance (cross-validated AUC 0.92 all data, 0.79 pretest). In the minimal-features model, we were able to retain high accuracy (cross-validated AUC 0.87 all data, AUC 0.80 pre-test) despite only including 4 questions, one of which was a symptom and three referring to potential contact with known infected individuals. Restricting the observed inputs to the 1,613 users ( 89 positive, 1,524 negative) who answered the survey in the 14 days 
medRxiv preprint doi: https://doi.org/10.1101/2020.06.09.20126813; this version posted June 11, 2020. The copyright holder for this preprint (which was not certified by peer review) is the author/funder, who has granted medRxiv a license to display the preprint in perpetuity. All rights reserved. No reuse allowed without permission.

prior to being tested limited the sample size and reduced the overall accuracy, but the relative performance of the models was similar (Fig. 3b).

The fact that a fraction of SARS-CoV-2(+) users report no symptoms or only less common symptoms (Fig. $2 \mathrm{~g}$ ) raises the possibility that many infected users might behave in ways that could spread disease, such as leaving home while unaware that they are infectious. In spite of widespread shelter-in-place orders during the sample period, we found extensive heterogeneity across the US in the fraction of users reporting leaving home each day, with $61 \%$ of the responses from April 24 - May 12 indicating the user had left home that day (Fig. 4a). The majority (77\%) of these users reported leaving for non-work reasons, including exercising; 19\% left for work (Fig. 4b). Of people who left home, a majority but not all users reported social distancing and using face protection (Fig. 4c). This incomplete shutdown, and lack of total social and physical protective measures, coupled with insufficient isolation of infected cases, may contribute to continued disease spread.

Given the large number of people leaving home each day, it is important to understand the behavior of people who are potentially infectious and therefore likely to spread SARS-CoV-2. To this end, we further analyzed the behavior of people both reporting to be PCR test $(+)$ or $(-)$. There was an abrupt large increase in users reporting staying home after receiving a positive test result (Fig. 4d,e). Many, but not all, PCR test(+) users reported staying home in the 2-7 days after their test date (7\% still went to work), whereas $23 \%$ of untested and $26 \%$ of PCR test(-) left for work (Fig. 4d,e). Similarly, 3\% of PCR test(+) users reported going to work without a mask, in contrast with untested (12.7\%) and PCR test(-) (10\%) users (Fig. 4f). Positive individuals reported coming into close contact with a median of 1 individual over 3 days in contrast to individuals who tested negative or were untested, who typically came in close contact with a median of 4 people within 3 days (Fig. 4g). Regression analysis suggested that healthcare workers (OR: 9.6, 95\% CI: [7.6, 12.1]) and other essential workers (OR: 7.0, 95\% CI: $[5.4,9.1])$ were much more likely to go to work after taking a positive or negative test, and PCR positive users were more likely to stay home (OR: 0.1, 95\% CI: [0.1, 0.2]) (Fig. 4h, Extended Data Table 12).

We find evidence among our users for several factors that could contribute to continued COVID19 spread despite widespread implementation of public health measures. These include a substantial fraction of users leaving their homes on a daily basis across the US, users who claim to not socially isolate or return to work after receiving a PCR test $(+)$, self-reports of asymptomatic, mildly symptomatic, or presymptomatic presentation, and a much higher risk of infection for people with within-household exposure. That said, we note several limitations of this study. The HWF user base is inherently a non-random sample of voluntary users of a smartphone app, and hence our results may not fully generalize to the broader US population. Our results are based on self-reported survey data, hence may suffer from misclassification bias - particularly those based on self-reported behaviors. Moreover, a relatively small percentage of the US population has been prioritized for PCR testing, so any analysis of test results (or tested users) may be subject to 
selection bias. While we have attempted to correct for these biases via the inverse probability weighting approach (Methods), some residual bias may persist if there remain some unobserved factors related to underlying disease status and receiving a test.

Although there is enormous economic pressure on states, businesses, and individuals to be able to return to work as quickly as possible, our findings highlight the ongoing importance of social distancing, mask wearing, large-scale testing of both symptomatic and asymptomatic people, and potentially even more rigorous 'test-trace-isolate' approaches ${ }^{37-40}$ as implemented in Massachusetts, New York, and New Jersey to bend the infection curve ${ }^{37-40}$. Applying predictive models on a population scale will be vitally important to provide an "early warning" system for timely detection of a second wave of infections in the US and for guiding an effective public policy response. At an individual level, our data show that adding information beyond symptoms, such as household and community exposure, occupation, and demographics, is vital for identifying infected individuals from self-reported data. As testing resources are expected to continue to be limited, HWF results could be used to identify which groups should be prioritized, or potentially to triage individuals for molecular testing based on predicted risk. HWF's integration of behavioral, symptom, exposure, and demographic data provides a powerful platform to address emerging problems in controlling infection chains, rapidly assist public health officials and governments with developing evidence-based guidelines in real time, and stop the spread of COVID-19.

\section{References}

1. Zhou, P. et al. A pneumonia outbreak associated with a new coronavirus of probable bat origin. Nature 579, 270-273 (2020).

2. Wölfel, R. et al. Virological assessment of hospitalized patients with COVID-2019. Nature 1-10 (2020). doi:10.1038/s41586-020-2196-x

3. Sanche, S. et al. High Contagiousness and Rapid Spread of Severe Acute Respiratory Syndrome Coronavirus 2. Emerg. Infect. Dis. J. 26, (2020).

4. Schuchat, A. Public Health Response to the Initiation and Spread of Pandemic COVID-19 in the United States, February 24-April 21, 2020. MMWR. Morb. Mortal. Wkly. Rep. 69, 551-556 (2020).

5. Kraemer, M. U. G. et al. The effect of human mobility and control measures on the COVID-19 epidemic in China. Science (80-. ). (2020). doi:10.1126/science.abb4218

6. Chen, H., Qian, W. \& Wen, Q. The Impact of the COVID-19 Pandemic on Consumption: Learning from High Frequency Transaction Data. SSRN Electron. J. (2020). doi:10.2139/ssrn.3568574

7. Worldometer. Coronavirus Cases. Worldometer 1-22 (2020). doi:10.1101/2020.01.23.20018549V2

8. Smolinski, M. S. et al. Flu near you: Crowdsourced symptom reporting spanning 2 influenza seasons. Am. J. Public Health 105, 2124-2130 (2015).

9. Segal, E. et al. Building an International Consortium for Tracking Coronavirus Health Status. 
medRxiv preprint doi: https://doi.org/10.1101/2020.06.09.20126813; this version posted June 11, 2020. The copyright holder for this preprint (which was not certified by peer review) is the author/funder, who has granted medRxiv a license to display the preprint in perpetuity.

All rights reserved. No reuse allowed without permission.

medRxiv 2020.04.02.20051284 (2020). doi:10.1101/2020.04.02.20051284

10. Menni, C. et al. Real-time tracking of self-reported symptoms to predict potential COVID-19. Nat. Med. 1-4 (2020). doi:10.1038/s41591-020-0916-2

11. Rossman, H. et al. A framework for identifying regional outbreak and spread of COVID-19 from one-minute population-wide surveys. Nature Medicine 26, 634-638 (2020).

12. Drew, D. A. et al. Rapid implementation of mobile technology for real-time epidemiology of COVID-19. Science (80-. ). eabc0473 (2020). doi:10.1126/science.abc0473

13. Hao, X. et al. Full-spectrum dynamics of the coronavirus disease outbreak in Wuhan, China: a 2 modeling study of 32,583 laboratory-confirmed cases. medRxiv (2020).

14. Verity, R. et al. Estimates of the severity of coronavirus disease 2019: a model-based analysis. Lancet Infect. Dis. 0, (2020).

15. Onder, G., Rezza, G. \& Brusaferro, S. Case-Fatality Rate and Characteristics of Patients Dying in Relation to COVID-19 in Italy. JAMA - Journal of the American Medical Association 323, 17751776 (2020).

16. Maxmen, A. Thousands of coronavirus tests are going unused in US labs. Nature 580, 312-313 (2020).

17. COVID-19 and the Potential Devastation of Rural Communities: Concern from the Southeastern Belts. Available at: https://deepblue.lib.umich.edu/handle/2027.42/154715. (Accessed: 17th May 2020)

18. Rader, B. et al. Geographic access to United States SARS-CoV-2 testing sites highlights healthcare disparities and may bias transmission estimates. J. Travel Med. taaa076, (2020).

19. How to Use the Data The COVID Tracking Project. Available at: https://covidtracking.com/about-data. (Accessed: 17th May 2020)

20. Coronavirus Disease 2019 (COVID-19). Available at: https://www.cdc.gov/coronavirus/2019ncov/symptoms-testing/symptoms.html.

21. Wei, W. E. et al. Presymptomatic Transmission of SARS-CoV-2 - Singapore, January 23-March 16, 2020. MMWR Morb Mortal Wkly Rep 69, 411-415 (2020).

22. Linton, N. M. et al. Incubation Period and Other Epidemiological Characteristics of 2019 Novel Coronavirus Infections with Right Truncation: A Statistical Analysis of Publicly Available Case Data. J. Clin. Med. 9, 538 (2020).

23. Li, R. et al. Substantial undocumented infection facilitates the rapid dissemination of novel coronavirus (SARS-CoV2). Science 368, 489-493 (2020).

24. Sutton, D., Fuchs, K., D’Alton, M. \& Goffman, D. Universal Screening for SARS-CoV-2 in Women Admitted for Delivery. N. Engl. J. Med. 1-2 (2020). doi:10.1056/nejmc2009316

25. Baggett, T. P., Keyes, H., Sporn, P.-C. N. \& Gaeta, J. M. Prevalence of SARS-CoV-2 Infection in Residents of a Large Homeless Shelter in Boston. JAMA - J. Am. Med. Assoc. E1-E2 (2020). doi:10.1001/jama.2020.6887

26. Kucirka, L. M., Lauer, S. A., Laeyendecker, O., Boon, D. \& Lessler, J. Variation in FalseNegative Rate of Reverse Transcriptase Polymerase Chain Reaction-Based SARS-CoV-2 Tests by Time Since Exposure. Ann. Intern. Med. (2020). doi:10.7326/m20-1495

27. Griffith, G., Morris, T. T., Tudball, M., Herbert, A. \& Mancano, G. Collider bias undermines our understanding of COVID-19 disease risk and severity Affiliations. 1-29 (2020).

28. Aylward, B. \& Liang, W. Report of the WHO-China Joint Mission on Coronavirus Disease 2019 (COVID-19). WHO-China Jt. Mission Coronavirus Dis. 2019 2019, 16-24 (2020).

29. Nishiura, H. et al. Closed environments facilitate secondary transmission of coronavirus disease 2019 (COVID-19). medRxiv 2-3 (2020).

30. He, X. et al. Temporal dynamics in viral shedding and transmissibility of COVID-19. Nat. Med. 26, 672-675 (2020).

31. Wang, Z., Ma, W., Zheng, X., Wu, G. \& Zhang, R. Household transmission of SARS-CoV-2. J. Infect. (2020). doi:10.1016/j.jinf.2020.03.040

32. Jing, Q.-L. et al. Household Secondary Attack Rate of COVID-19 and Associated Determinants. 
medRxiv preprint doi: https://doi.org/10.1101/2020.06.09.20126813; this version posted June 11, 2020. The copyright holder for this preprint

(which was not certified by peer review) is the author/funder, who has granted medRxiv a license to display the preprint in perpetuity.

All rights reserved. No reuse allowed without permission.

medRxiv 2020.04.11.20056010 (2020). doi:10.1101/2020.04.11.20056010

33. Bi, Q. et al. Epidemiology and transmission of COVID-19 in 391 cases and 1286 of their close contacts in Shenzhen, China: a retrospective cohort study. Lancet Infect. Dis. 3099, 1-9 (2020).

34. County, S. et al. High SARS-CoV-2 Attack Rate Following Exposure at a Choir Practice - . 69, 606-610 (2020).

35. Gibbins, J. D. et al. COVID-19 Among Workers in Meat and Poultry Processing Facilities —. 69, 557-561 (2020).

36. McMichael, T. M. et al. Epidemiology of Covid-19 in a Long-Term Care Facility in King County, Washington. N. Engl. J. Med. 1-7 (2020). doi:10.1056/NEJMoa2005412

37. Pan, A. et al. Association of Public Health Interventions with the Epidemiology of the COVID-19 Outbreak in Wuhan, China. JAMA - J. Am. Med. Assoc. 02115, 1915-1923 (2020).

38. Clark, G. et al. COVID-19 pandemic: some lessons learned so far. (UK House of Commons Science and Technology Committee, 2020).

39. Finberg, H. V. Ten Weeks to Crush the Curve. N. Engl. J. Med. 382, e37 (2020).

40. Kim, J. Y. It's Not Too Late to Go on Offense Against the Coronavirus. New Yorker (2020). Available at: https://www.newyorker.com/science/medical-dispatch/its-not-too-late-to-go-onoffense-against-the-coronavirus. (Accessed: 27th May 2020) 


\section{Figures}

a

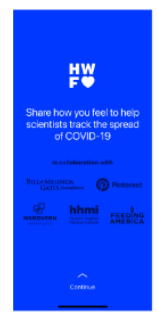

The How We Feel app
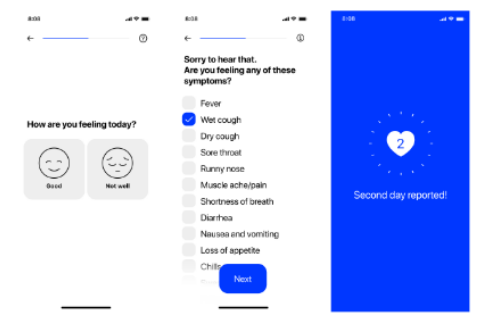

C

Demographic

Age

Location

Race/ethnicity

Gender

Occupation

Household size

Behavior

Left home today

Wear mask

Socially distance

Food source
Medical

Pre-existing conditions

Feel well/not well

Symptoms

COVID test results

Contact with infected

Hours of sleep

Smoking

Sentiment

Feel safe to return to work Skipped doctors appointments

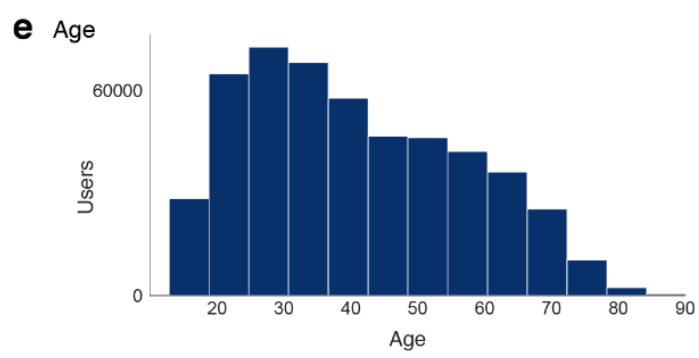

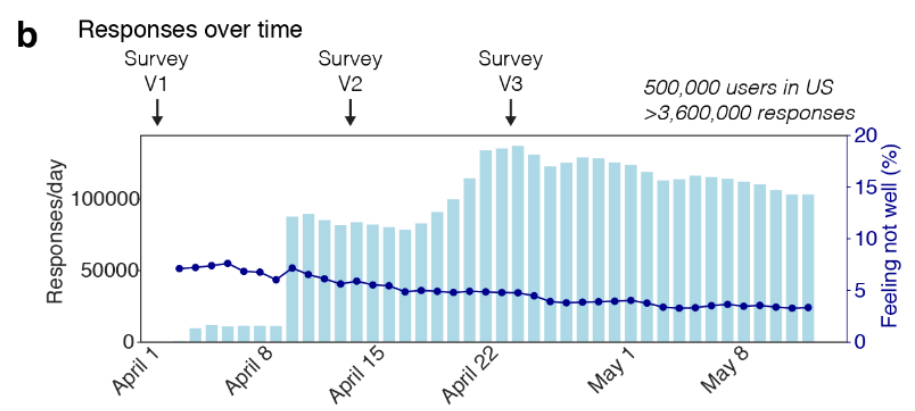

d Users by state

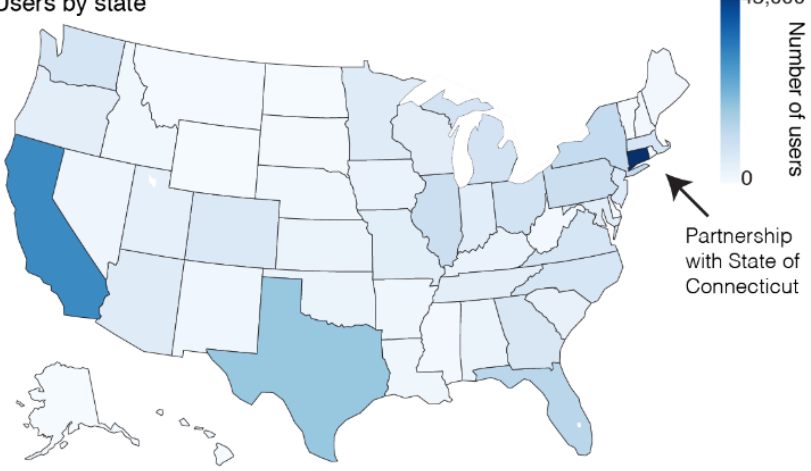

f Gender

g Race/ethnicity

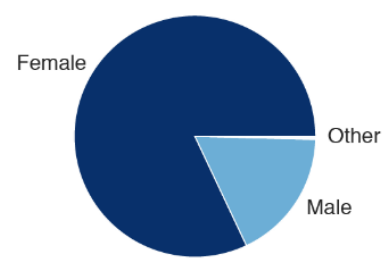

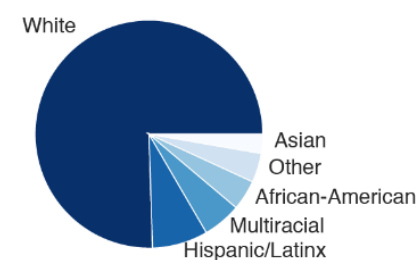

Figure 1: The How We Feel Application and User Base.

a, The How We Feel (HWF) app: longitudinal tracking of self-reported COVID-19-related data. b, Responses over time, as well as percentage of users reporting feeling unwell, with releases of major updates to survey indicated. c, Information collected by the HWF app. d, Users by state across the United States. e, Age distribution of users. Note: users had to be older than 18 to use the app. f, Distribution of self-reported sex. g, Distribution of self-reported race or ethnicity. Users were allowed to report multiple races. "Multiracial" = the user indicated more than one category. "Other" includes American Indian/Alaskan Native and Hawaiian/Pacific Islander, as well as users who selected "Other". 
medRxiv preprint doi: https://doi.org/10.1101/2020.06.09.20126813; this version posted June 11, 2020. The copyright holder for this preprint (which was not certified by peer review) is the author/funder, who has granted medRxiv a license to display the preprint in perpetuity.
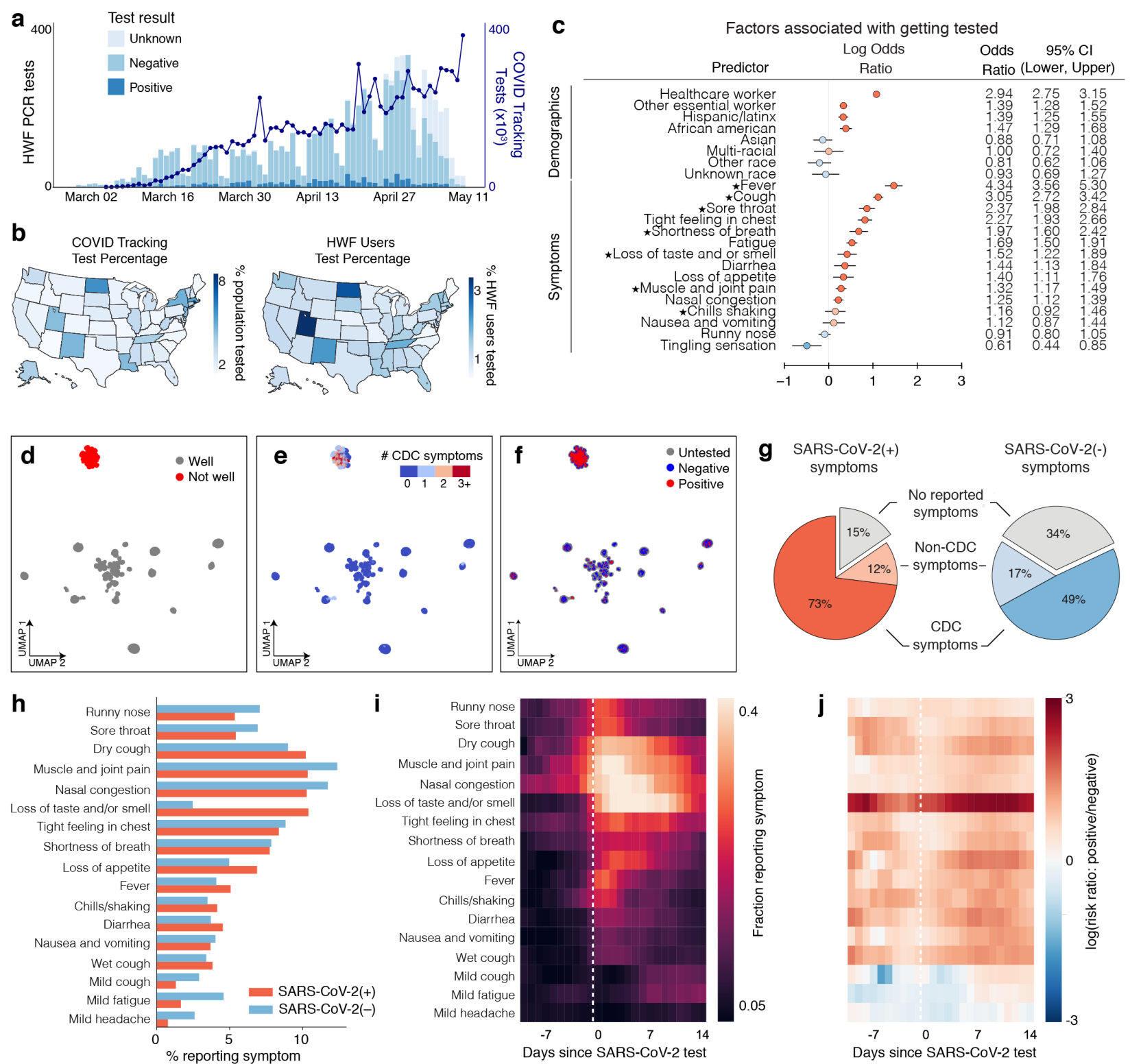

\section{Figure 2: SARS-CoV-2 PCR Testing and Symptoms.}

a, Stacked bar plot of user-reported test results over time, overlaid with official number of tests across US based on COVID Tracking Project data. b, Left: Map of per-capita test rates across the United States. Right: Map of COVID-19 tests per number of users by state. c, Associations of professions and symptoms with receiving a SARS-CoV-2 PCR test, adjusted for demographics and other covariates (Methods). Common symptoms listed by the CDC are starred. d-f, UMAP visualization of 667,651 multivariate symptom responses among HWF users that reported at least one symptom. Coloring indicates: $\mathbf{d}$, responses according to users feeling well; $\mathbf{e}$, the reported number of COVID-19 symptoms listed by the CDC; and $\mathbf{f}$, the COVID19 test result among tested users. g, Proportion of positive COVID-19 patients (red) and negative COVID19 patients (blue) experiencing either CDC-common symptoms (dark), only non-CDC symptoms (light), or no symptoms (grey) on the day of their test. $\mathbf{h}$, Histogram of reported symptoms among COVID-19 
medRxiv preprint doi: https://doi.org/10.1101/2020.06.09.20126813; this version posted June 11, 2020. The copyright holder for this preprint (which was not certified by peer review) is the author/funder, who has granted medRxiv a license to display the preprint in perpetuity.

All rights reserved. No reuse allowed without permission.

tested users. i, Longitudinal self-reported symptoms from users that tested positive for COVID-19. Dates are centered on the self-reported test-date. $\mathbf{j}$, Ratio of symptoms comparing users that test positive versus test negative for COVID-19. 
medRxiv preprint doi: https://doi.org/10.1101/2020.06.09.20126813; this version posted June 11, 2020. The copyright holder for this preprint (which was not certified by peer review) is the author/funder, who has granted medRxiv a license to display the preprint in perpetuity. All rights reserved. No reuse allowed without permission.
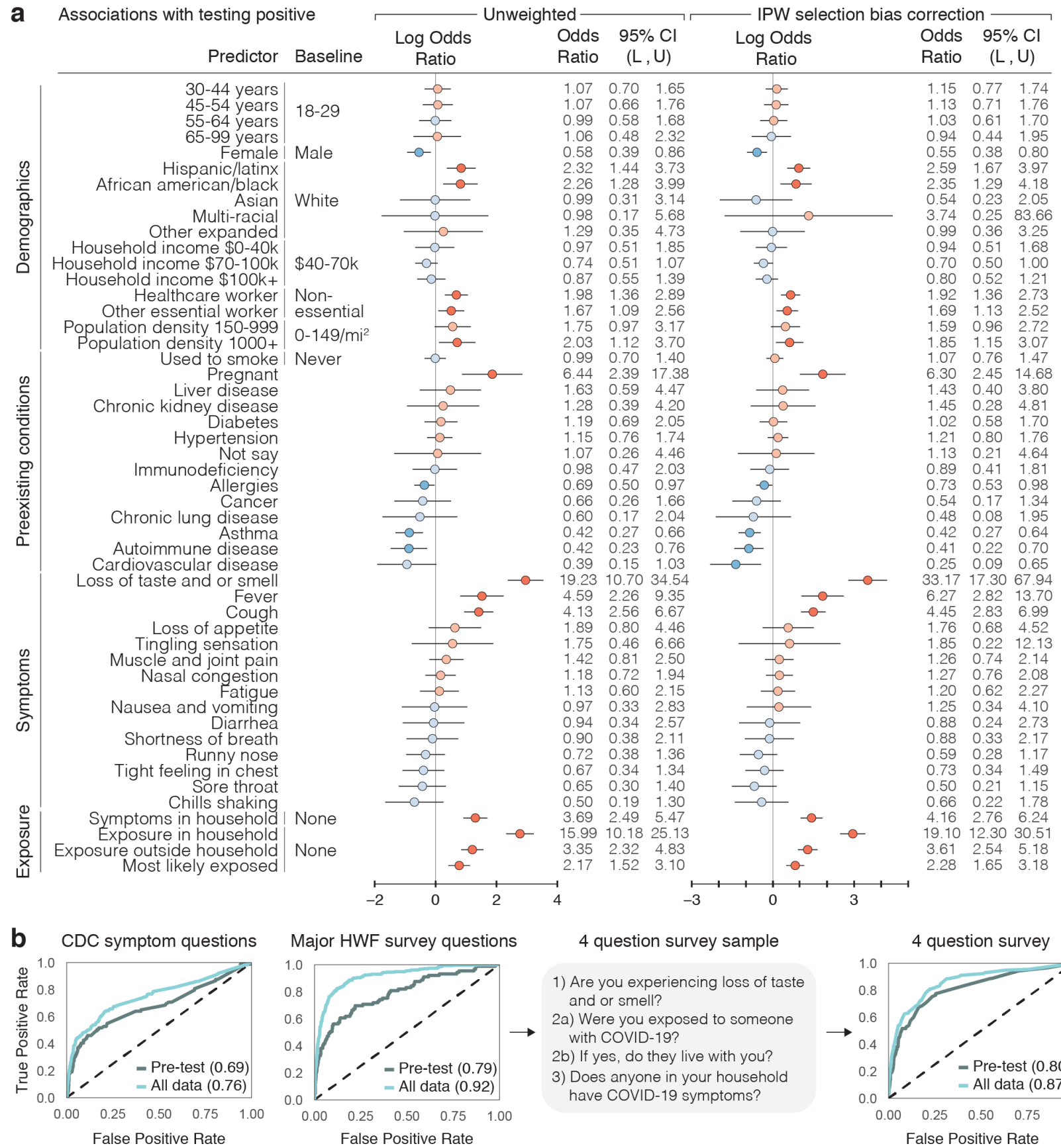

4 question survey sample

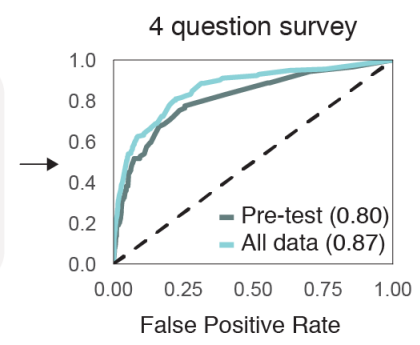

\section{Figure 3: SARS-CoV-2 PCR Test Result Associations and Predictions.}

a, Factors associated with respondents receiving and reporting a positive test result, as determined through logistic regression. Left: results from unweighted model. Right: results from model incorporating propensity scores via inverse probability weights (IPW). Reference categories are indicated where relevant, and when not indicated, the reference is not having that specific feature. Log odds ratios and their confidence intervals are plotted, with red indicating positive association and blue indicating negative association. Darker colors indicate confidence intervals that do not cover 0. Population density and 
medRxiv preprint doi: https://doi.org/10.1101/2020.06.09.20126813; this version posted June 11, 2020. The copyright holder for this preprint (which was not certified by peer review) is the author/funder, who has granted medRxiv a license to display the preprint in perpetuity.

All rights reserved. No reuse allowed without permission.

neighborhood household income were imputed from user demographics. $\mathrm{L}=$ lower bound, $\mathrm{U}=$ upper bound of $95 \%$ confidence intervals. $\mathbf{b}$, Prediction of positive test results using \pm 2 weeks of data from test date, using 5-fold cross validation, shown as receiver operating characteristic (ROC) curves. The XGBoost model was trained on different subsets of questions: CDC Symptom Questions = using just the subset of COVID19 symptoms listed by the CDC. All Survey Questions = using the entire survey. 4 Question survey $=$ using a reduced set of 4 questions that were found to be highly predictive. Numerical values are AUC $=$ area under the ROC curve. 


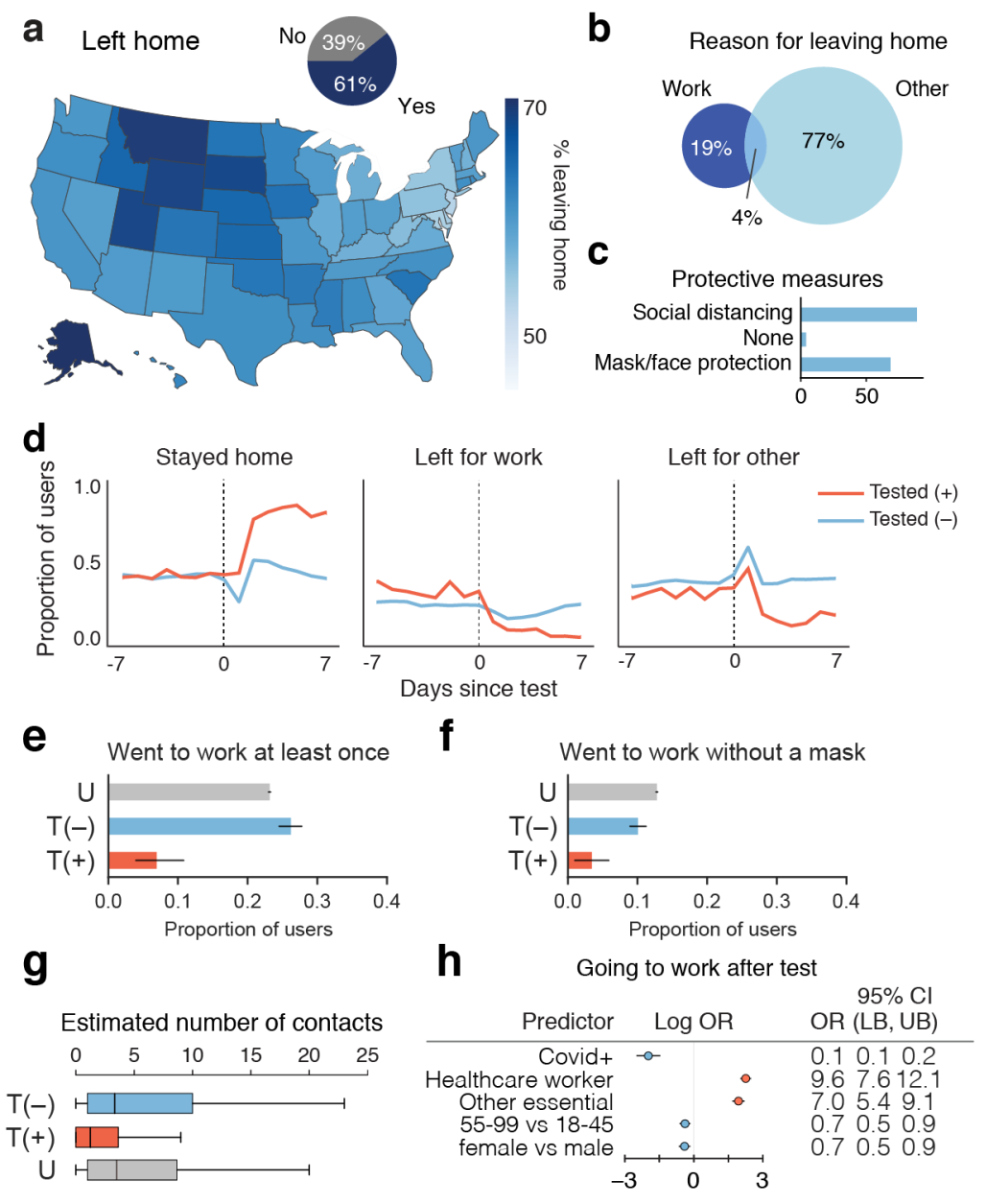

\section{Figure 4: Behavioral Factors Potentially Contributing to COVID-19 Spread.}

a, Proportion of responses indicated users leaving home across US (map) or overall (inset pie chart). b, Percentage of responses of users reporting work or other reason for leaving home. c, Reported protective measures taken per response taken by users upon leaving home. d, Time course of proportion of SARSCoV-2 PCR tested positive $(+)$ or negative $(-)$ users staying home, leaving for work, and leaving for other reasons. e-f, Proportion SARS-CoV-2 PCR tested $(+)$ or $(-)$, or untested (U), going to work (e), going to work without a mask (f) in the 2-7 days post test for $\mathrm{T}=$ tested, or 3 weeks since last check in for $\mathrm{U}=$ untested. Healthcare workers and other essential workers are compared to non-essential workers as the baseline. g, Average reported number of contacts per 3 days in the 2-7 days after their test date. OR= odds ratio, $\mathrm{LB}=$ lower bound, $\mathrm{UB}=$ upper bound, $\mathrm{CI}=$ confidence interval, $\mathrm{T}=$ tested, $\mathrm{P}=$ positive, $\mathrm{U}=$ untested. $\mathbf{h}$, Logistic regression analysis of factors contributing to users going to work in the 2-7 days after their COVID-19 test. 


\section{Additional Material}

\section{Acknowledgements:}

The How We Feel Project would like to thank operational volunteers Ari Simon, Ricki Seidman, Arun Ranganathan, Celie O’Neil-Hart, Debbie Adler, Divya Silbermann, Jack Chou, Lother Determann, Mark Terry, Rhiannon Macrae, Robert Barretto, Ron Conway, Sid Shenai, Tony Falzone, and Yurie Shimabukuro. We would also like to thank Andrew Tang for graphic design support. We are grateful to the HWF participants who took our survey and allowed us to share our analysis.

\section{Author contributions:}

W.E.A., H.A.-T., J.B., X.J., G.M., A.S., R.R., N.N., M.K. contributed to analysis. W.E.A., H.A.-T., J.B., X.J., and G.M., performed the majority of data cleaning, analysis, figures production, and wrote the manuscript with F.Z. and X.L. A.S. and R.R. performed household transmission and symptom type analysis. W.E.A. coordinated the analysis effort. A.P., C.D., A.K., J.I., T.H., E.C., C.L., M.C., H.B., W.L., R.M., R.P, B.S. designed and implemented the How We Feel application. B.C., M.T., J.O., C.S.G., O.S., G.K., and D.R.C. designed the survey, and provided feedback on app design and analysis. B.S. and F.Z. initiated the project. F.Z. and X.L. supervised all aspects of the work.

\section{Funding:}

The How We Feel Project is a non-profit corporation. Funding and in-kind donations for the How We Feel Project came from Ben and Divya Silbermann, Feng Zhang and Yufen Shi, Lore Harp McGovern, David Cheng, Ari Azhir, and Kyung H. Yoon, and the Bill \& Melinda Gates Foundation. X.L. acknowledges support from Harvard University and NCI R35-CA197449-05. F.Z. is supported by the Howard Hughes Medical Institute, the McGovern Foundation, and James and Patricia Poitras and the Poitras Center.

\section{Competing Interests:}

The authors declare no competing financial interests.

\section{Data and materials availability:}

This work used data from the How We Feel project (http://www.howwefeel.org/). The software used in analysis will be made available on Github upon final publication of the paper. Researchers with IRB approval to perform research involving human subjects can apply to obtain access to data used in the analysis. 